\title{
Sex composition and group performance in a visual signal detection task
}

\author{
DAVID E. CLEMENT and JOSEPH J. SCHIERECK, JR. \\ University of South Florida, Tampa, Florida 33620
}

\begin{abstract}
Forty-eight Ss (24 males, 24 females) were run in groups of 4 on 720 trials of a 16-alternative forced-choice visual signal detection task requiring both individual and group decisions. Four types of groups were formed: all male, all female, mixed-alternate (seated in the order male-female-male-female), and mixed-adjacent (seated in the order male-male-female-female). There were no differences in performance between all-male and all-female groups, but mixed-sex groups had poorer performance, with mixed-adjacent groups having significantly lower sensitivity than homogeneous-sex groups. The results were interpreted as indicating that heterogeneity of groups with regard to sex interacts with seating pattern in affecting performance, probably due to the formation of information coalitions between like-sex group members when the seating pattern encourages such coalitions.
\end{abstract}

A programmatic series of studies of visual signal detection determined that scanning strategies were similar for most individuals, both male and female (Clement \& Hosking, 1971; Clement \& Schiereck, 1971). It also was found that group performance in four-person groups was superior to individual performance, but was not as good as predicted by a model in which information from individuals was combined in an independent fashion (Clement, 1973). Although this last study used only male Ss, prior findings indicated that the task was not sex-specific, and thus groups of female Ss would be expected to perform in a similar manner. However, this speculation was not subjected to empirical test. In extending investigation to the possible effects of $S$ sex on group performance, three types of groups could be considered: all-male, all-female, and mixed groups. Mixed groups, in turn, could contain differing proportions of males and females and could differ as well in terms of the seating patterns (proximity relations) among males and females in the group.

Mixed groups, by definition, are heterogeneous in nature. Studies of group homogeneity and its effect on performance generally have dealt with personaity or prior performance factors (e.g., Hoffman, 1959; Goldman, Dietz, \& McGlynn, 1968), though a few have included sex as a variable in studies of such things as problem-solving tasks (Hoffman \& Maier, 1961; Kent \& McGrath, 1969) and jury deliberations (Strodtbeck \& Mann, 1956). In the latter studies, the usual interpretation has been that obtained sex differences evolved from differences in motivation and culturally determined role expectations-perhaps of more competitiveness on the part of males (e.g., Uesugi \& Vinacke, 1963). No evidence has been obtained that either males or females typically perform in a superior fashion on nonsex-specific tasks. Aside from such studies, sex composition of groups has been used infrequently as a variable in small group performance studies. It might be expected that mixed groups would show some of the effects previously attributed to group heterogeneity, but the effect of sex heterogeneity per se probably would be much less than that of heterogeneity due to personality and other differences.

One factor in small group performance receiving more study has been that of proximity or seating relationships. Such studies usually have focused on leadership roles and their development (e.g., Davenport, Brooker, \& Munro, 1971). Those members of a group who are seated near each other might be expected to communicate more (Hare \& Bales, 1963), and this could lead to increased cooperative behavior as well as to solidification of leadership roles (Sommer, 1961). All-male and all-female groups would not differ in the respect that there would be no sex-related proximity relationships to affect communications and leadership functions. However, mixed groups might be expected to show some such effects, particularly if the seating arrangement were one which tended to emphasize like-sex grouping.

Another potential factor which might influence small group performance would be coalition formation by sex in mixed groups. In cooperative kinds of tasks, the formation of coalitions is rather unexpected. However, if coalitions were formed, the task would take on some aspects of a competitive rather than a cooperative situation, and performance might be expected to suffer (e.g., Deutsch, 1949). Such coalitions might form as a result of sex-role differentiation (Strodtbeck \& Mann, 1956) or some other sex-related behavior which influences communications-object choices (Parten, 1933; Smith, 1944; James, 1951). Coalitions based upon S sex would be more likely to form when such things as proximity or seating patterns would encourage them (Hare, 1962). For example, a four-person mixed-sex group with two males and two females could be seated in the order male-male-female-female or in the order male-female-male-female. The former arrangement would be expected to emphasize and encourage sex grouping.

This study compared the performances of four types 
Table 1

Proportion Correct $\left(\mathbf{P}_{\mathcal{C}}\right)$ for Each Target Location for Each Type of Group

\begin{tabular}{lcrrr}
\hline & \multicolumn{4}{c}{ Location } \\
\cline { 2 - 5 } & 1 & 2 & 3 & 4 \\
\hline All Males & .73 & 1.00 & 1.00 & .73 \\
All Females & .77 & .83 & .93 & .43 \\
M-F-M-F & .60 & .70 & .90 & .37 \\
M-M-F-F & .13 & .40 & .63 & .40 \\
\hline & 5 & 6 & 7 & 8 \\
\hline All Males & .77 & .87 & 1.00 & .97 \\
All Females & .93 & 1.00 & 1.00 & .73 \\
M-F-M-F & .93 & 1.00 & 1.00 & .57 \\
M-M-F-F & .77 & 1.00 & 1.00 & .60 \\
\hline & 9 & 10 & 11 & 12 \\
\hline All Males & .73 & .70 & .67 & .63 \\
All Females & .93 & .80 & .93 & .60 \\
M-F-M-F & .90 & .93 & .83 & .40 \\
M-M-F-F & .80 & .87 & 1.00 & .57 \\
\hline & 13 & 14 & 15 & 16 \\
\hline All Males & .17 & .27 & .17 & .03 \\
All Females & .23 & .27 & .27 & .27 \\
M-F-M-F & .07 & .23 & .30 & .17 \\
M-M-F-F & .00 & .27 & .13 & .07 \\
\hline
\end{tabular}

of groups, each involving four Ss: (1) all male; (2) all female; (3) mixed-alternate, with the seating pattern male-female-male-female; and (4) mixed-adjacent, with the seating pattern male-male-female-female. The performance task was the 16-alternative forced-choice visual signal detection task used previously (Clement \& Hosking, 1971, et seq.). The performance comparisons of interest were those of all-female with all-male groups and those of each of the two types of mixed groups with each other and with the homogeneous-sex groups.

\section{METHOD}

Stimuli were on $35-\mathrm{mm}$ slides and were presented by means of a Lafayette two-field projection tachistoscope, with a blank frame identical in size to the stimulus frame projected at all times other than during stimulus presentation. Each stimulus consisted of a square field containing 16 black outline circles in a 4 by 4 array. Fifteen of the circles contained a black outline "upright" equilateral triangle (base down), and the 16th circle contained the target, an "inverted" equilateral triangle (base up) For purposes of analysis and for ease of recording during the experiment, the circles were assigned consecutive numbers, beginning with the upper left corner and progressing from left to right in each row. The data in Tables 1 and 2 are presented in a spatial arrangement similar to that of the original target locations. Ss were seated approximately $3.4 \mathrm{~m}$ from the projection screen, and the projected stimulus frame measured $30.5 \mathrm{~cm}$ on a side. Each projected circle measured $4.6 \mathrm{~cm}$ in diam, containing a centered equilateral triangle, $2 \mathrm{~cm}$ on a side. The visual angle subtended by the 16 circles was approximately $4 \mathrm{deg}$, with each circle subtending an angle of about $48 \mathrm{~min}$ and each triangle subtending an angle of about $20 \mathrm{~min}$. The four Ss in each group were seated side by side on a slightly curved line, so that each had an approximately normal view of the projection screen. Partitions between the work spaces for the $\mathrm{Ss}$ allowed them to see each other but screened the writing surface for each $S$ from the view of other Ss. A small diagram in each S's booth contained the 16 target locations with the appropriate identification number in each, and the meaning of the numbers used as confidence ratings, for reference during the experiment. Ss were assigned randomly to the four chairs on Day l (with exceptions noted below) and retained the same chair on subsequent days.

Forty-eight $S_{s}(24$ males, 24 females) were used in the experiment. run in groups of 4 . Groups $1-4$ contained all males Groups 5-8. all females: Groups 9-10. males and females seated in the pattern MFMF (mixed-alternate); Groups 11-12, males and females seated in the pattern MMFF (mixed-adjacent). Each group was run for a total of 720 trials. The experiment extended through five 1-h sessions. sessions occurring at least 3 days apart. On Day 1 and Day 5, all groups were given "individual" instructions and were run on 240 trials per day. Ss were instructed to "try to pay attention to all 16 possible target locations," were told that the target was placed in a random fashion, and were given a brief description of the meaning of random ordering. Instructions on intervening days referred to the instructions for Day 1 with modifications noted below. Each trial consisted of a presentation of a stimulus slide for $200 \mathrm{msec}$, with about $6 \mathrm{sec}$ between trials. During the intertrial interval, each $S$ recorded the location of the target (using the appropriate location number), together with a confidence rating for the judgment. Confidence ratings consisted of the numbers $1-5$, with 1 meaning a complete guess, 5 meaning complete certainty. and intermediate numbers referring to intermediate levels of confidence. On Days 2-4, 80 trials were run per day, with four groups $(1,2,5,6)$ given "group only" instructions and all other groups given "individual plus group" instructions. "Individual plus group" instructions required each $S$ to make individual responses as on Day 1, then after all $S s$ in the group had recorded their responses on each trial, the members of the group communicated with each other in reaching a group decision concerning the location of the target and the group confidence concerning this decision. This group decision was recorded by the $\mathrm{E}$. No instructions were given as to how this decision was to be reached, and no time limits were placed upon the groups. Thus, stimuli were presented as during Day 1, but the intertrial interval varied from trial to trial. "Group only" instructions required the same discussion and group decision, but no individual decisions were recorded by the $S$ s. On all days, brief rest periods were given after blocks of 40 trials. Stimuli were presented in a pseudorandom order, with the target occurring in each location five times in consecutive series of 80 trials and thus 45 times in the experiment. Five practice trials were run prior to data collection on Day 1 to assure appropriate interpretation of the instructions by the Ss. There was no feedback given to the Ss during the experiment.

\section{RESULTS}

Proportion correct $\left(\mathrm{P}_{\mathrm{c}}\right)$ was obtained for each target location and for all target locations combined for consecutive blocks of 240 trials and for all trials combined. Classifications for which $P_{c}$ was obtained included individual $S$ s, sex of $S$, all $S s$ combined, each group, each type of group, and all groups combined. $P_{c}$ ranged from .18 to .56 for individual $S s$ across all trials and target locations. Table 1 contains $P_{c}$ values for each type of group for each target location across all group decision trials. Data include only those two groups in each type of group which received "individual plus group" instructions. Proportion of hits (correct identifications of target locations) and proportion of 
false alarms (trials on which a target location was specified when, in fact, that target location was not correct) were calculated for each target location and for all target locations combined for the same classifications as described above. In performing these calculations. values near .00 and near 1.00 were rounded toward more central values so that a cumulative table for the normal distribution could be used to obtain $d^{\prime}$ values (the maximum standard deviation value allowed was 3.29 ). Values of $\mathrm{d}^{\prime}$ ranged from .71 to 2.04 for individual Ss across all trials and target locations; $\mathrm{d}^{\prime}$ ranged fiom .59 to 2.39 for individual Ss across all target locations for each block of 240 trials. Table 2 contains d' values for each type of group for each target location across all group decision trials. Data are from the same groups as in Table 1.

The comparisons of primary interest were those of mixed-sex groups with the homogeneous-sex groups on group performance. The all-male and all-female groups combined $\left(\mathrm{d}^{\prime}=3.10\right)$ did not differ significantly in overall detectability from the mixed-alternate groups ( $\mathrm{d}^{\prime}$ $=2.82)[\mathrm{t}(3)=2.17]$. However, the mixed-adjacent groups $\left(\mathrm{d}^{\prime}=2.58\right)$ were significantly lower in detectability index than were the male and female groups combined $[\mathrm{t}(3)=4.22 . \mathrm{p}<.05]$. The types of mixed-sex group did not differ significantly from each other, and detectability for the all-male groups $\left(\mathrm{d}^{\prime}=\right.$ 3.08) was almost identical to that for the all-female groups $\left(\mathrm{d}^{\prime}=3.12\right)$.

The two all-male and two all-female groups given "group only" instructions were included so that comparisons could be made between this experiment and the prior study using only male Ss (Clement, 1973). For complete comparison, data for the two types of pseudogroup (normative models) used in the prior experiment were calculated for Ss in this experiment. The details of calculation may be found in Clement (1973) and essentially result in one pseudogroup formed by using a logical, majority-vote algorithm for obtaining group responses from individual responses and a second pseudogroup formed by combining d's for individual Ss to obtain a "group" $d$ '. Analysis of variance for these data yjelded the same results as in the prior study, with no significance of the terms involving sex of $S$ or type of group (homogeneous or mixed). Terms which proved significant may be found by referring to the earlier study (Clement. 1973). Comparison of the male and female groups receiving "group only" instructions with all groups receiving "individual plus group" instructions replicated the findings of Clement (1973). That is, Ss in groups given "individual plus group" instructions improved more from the first to the third trial block than did Ss in groups receiving "group only" instructions, but the improvement was approximately the same for the former from the first to the second trial block as for the latter from the first to the third trial block. This confirmed the previous finding that the improvement in individual $d$ 'value was a function of the
Table 2

Detectability Index for Each Target Location for Each Type of Group

\begin{tabular}{lcccc}
\hline & \multicolumn{4}{c}{ Location } \\
\cline { 2 - 5 } & 1 & 2 & 3 & 4 \\
\hline All Males & 2.83 & 6.30 & 6.30 & 3.12 \\
All Females & 2.90 & 3.16 & 3.57 & 2.06 \\
M-F-M-F & 2.56 & 2.60 & 4.11 & 2.22 \\
M-M-F-F & 1.76 & 1.97 & 3.67 & 1.95 \\
\hline & 5 & 6 & 7 & 8 \\
\hline All Males & 2.74 & 2.59 & 5.95 & 4.90 \\
All Females & 4.30 & 5.10 & 5.26 & 3.92 \\
M-F-M-F & 4.06 & 5.05 & 4.84 & 2.18 \\
M-M-F-F & 3.50 & 5.02 & 4.71 & 1.86 \\
\hline & 9 & 10 & 11 & 12 \\
\hline All Males & 2.44 & 2.06 & 2.44 & 2.76 \\
All Females & 3.92 & 2.54 & 3.34 & 2.68 \\
M-F-M-F & 3.12 & 3.18 & 2.75 & 1.60 \\
M-M-F-F & 2.48 & 3.60 & 4.74 & 1.88 \\
\hline & 13 & 14 & 15 & 16 \\
\hline All Males & .84 & 1.85 & 1.66 & .58 \\
All Females & 1.23 & 1.52 & 2.01 & 2.30 \\
M-F-M-F & 1.32 & 2.09 & 1.92 & 1.60 \\
M-M-F-F & .00 & 1.94 & 1.64 & .62 \\
\hline
\end{tabular}

number of trials in which an individual response was required and recorded by the $S$ and was relatively unaffected by group practice. In brief, improvement in individual responses depended upon individual practice.

The confidence ratings were used to break ties in obtaining sensitivity values under one of the normative group models and also to provide an empirical test of the equal-variance Gaussian assumptions underlying the use of normal distribution tables to obtain d'. The normal, equal-variance assumption was a reasonable one, with plots of hits vs false alarms on double probability paper being linear and not too discrepant from a unit slope.

Observation of the discussion of all groups during trials in which they made group decisions indicated that the groups consistently used individual strategies, with discessions focusing on the relative certainty of different Ss when conflicts arose among individual decisions. The observations of individual groups did not indicate that males and females in the mixed groups behaved in a different fashion than did those in the homogeneous groups; only the data analysis of the detectability indexes demonstrated the difference.

The ordinal consistency of $d^{\prime}$ as a function of target location was reasonably high across classifications of $\mathrm{Ss}$ and groups and similar to that obtained previously (Clement, 1973; Clement \& Hosking, 1971: Clement \& Schiereck, 1971). Spearman's rho for rank order of sensitivity among target locations was higher than 80 for all comparisons between individual $S s$ and between .50 and .90 for comparisons between groups. 


\section{DISCUSSION}

Results with all-male groups replicated previous findings of increased sensitivity with group performance compared to individual performance, with less increase in sensitivity than would be predicted by independent combination of the sensitivity values for individual members of the group. Results with all-female groups did not differ from those for all-male groups, indicating that the task is nonsex-specific with regard to group performance as well as with regard to individual performance. The performance of all-male and all-female groups in this study was similar in all respects (scanning strategies, relative sensitivity to target location, etc.) to prior data (Clement, 1973).

Mixed-sex groups had less sensitivity to targets than did homogeneous-sex groups, with mixed-adjacent groups performing poorer than mixed-alternate groups (although only the difference between mixed-adjacent and homogeneous-sex groups was significant). Any model based upon combining information from several observers predicts that sensitivity of such a "group" increases with increasing number of independent observers. Thus, a decrement in performance for a group of given size would indicate that the group was performing as though it consisted of fewer members. In this experiment, the decrement in performance seemed clearly related to factors influencing like-sex clustering or coalition formation, such as seating pattern. Alternate-sex seating patterns appeared to reduce the likelihood of like-sex coalitions forming, while the adjacent-sex seating pattern appeared to increase this likelihood. An interesting facet of the results is that the performance data were the only indication that some kind of coalition was formed. Observation of the discussions of groups in reaching a group decision failed to indicate a trend toward coalition formation or decreased communication among the members of any group, including the mixed-adjacent groups. The interpretation of such performance differences as resulting from coalition formation of course is debatable. It could have been that effective communication occurred only between members of the same sex, thus reducing the amount of information available for "group" decisions, with no implication of overt functional coalitions being formed. Again, given the observational data, a distinction would have to be made between "communication" and "effective communication." Discussions were brief and seemed to adhere to the open communication pattern suggested by the instructions. Thus, communication as "overt talking" differed from communication as "sharing and using information." There was no evidence that the overt communication pattern was anything other than one with open channels among all group members for any kind of group. With no direct observations of coalition-like behavior, it might be better to refer to the formation of "information coalitions" in describing the results.

Any alternative explanation other than one based upon sex characteristics and seating pattern is not viable, as such an explanation would predict the same decrement in performance for all mixed-sex groups or possibly for all groups including the homogeneous-sex groups. Thus, a reasonable conclusion is that heterogeneity of groups as regards sex may not be sufficient to produce any information coalitions; however, a combination of heterogeneity of sex and a seating pattern which emphasizes the possibility of like-sex coalitions does lead to the formation of such information coalitions and concomitant reduced performance. Future research with small group performance on tasks such as this can safely ignore sex differences between homogeneous-sex groups, but must consider sex differences together with spatial relations among the members of the group when dealing with heterogeneous-sex groups.

In summary, this visual signal detection task was shown to be nonsex-specific, with similar performance between all-male groups and all-female groups. However, it was shown that heterogeneity of groups with regard to sex interacts with seating pattern in affecting performance, probably due to the formation of information coalitions between like-sex group members when the seating pattern encourages such coalitions.

\section{REFERENCES}

Clement, D. E. Group performance in a visual signal detection task. Human Factors, 1973, 15, 43-52.

Clement, D. E., \& Hosking, K. E. Scanning strategies and differential sensitivity in a visual signal detection task: Intrasubject reliability. Psychonomic Science, 1971, 22, 323-324.

Clement, D. E., \& Schiereck, J. J. Scanning strategies and differential sensitivity in a visual signal detection task: Intersubject consistency. Psychonomic Science, 1971, 23, 385-387.

Davenport, W. G., Brooker, G., \& Munro, N. Factors in social perception: Seating position. Perceptual \& Motor Skills, 1971, 33, 747-752.

Deutsch, M. An experimental study of the effects of cooperation and competition upon group process. Human Relations, 1949, $2,199-231$

Goldman, M., Dietz, D. M., \& McGlynn, A. Comparison of individual performance related to heterogeneous-wrong responses, size, and patterns of interaction. Psychological Reports, 1968, 23, 459-465.

Hare, A. P. Handbook of small group research. New York: Free Press, 1962.

Hare, A. P., \& Bales, R. F. Seating position and small group interaction. Sociometry, 1963, 26, 480-486.

Hoffman, L. R. Homogeneity of member personality and its effect on group problem-solving. Journal of Abnormal \& Social Psychology, 1959, 58, 27-32.

Hoffman, L. R., \& Maier, N. R. F. Sex differences, sex composition, and group problem solving. Journal of Abnormal \& Social Psychology, 1961, 63, 453-456.

James, J. Clique organization in a small industrial plant. 
Research Studies, State College, Washington, 1951, 19, Sommer, R. Leadership and group geography. Sociometry, 1961, 125-130.

Kent, R. N., \& McGrath, J. E. Task and group characteristics as factors influencing group performance. Journal of Experimental Social Psychology, 1969. 5, 429-440.

Parten, M. B. Social play among preschool children. Journal of Abnormal \& Social Psychology, 1933, 28, 136-147.

Smith, M. Some factors in the friendship selections of high school students. Sociometry, 1944, 7, 303-310. $24,99-110$.

Strodtbeck, F. L., \& Mann, R. D. Sex role differentiation in jury deliberations. Sociometry, 1956, 19, 3-11.

Uesugi, T. K., \& Vinacke, W. E. Strategy in a feminine game. Sociometry, 1963, 26, 75-88.

(Received for publication December 1, 1972; accepted January 31,1973 .) 